\title{
Simplicidad en la Administración pública y mejoramiento de la democracia
}

Simplicity in the Public Administration and improvement of democracy

Simplicidade na administração pública e melhoria da democracia
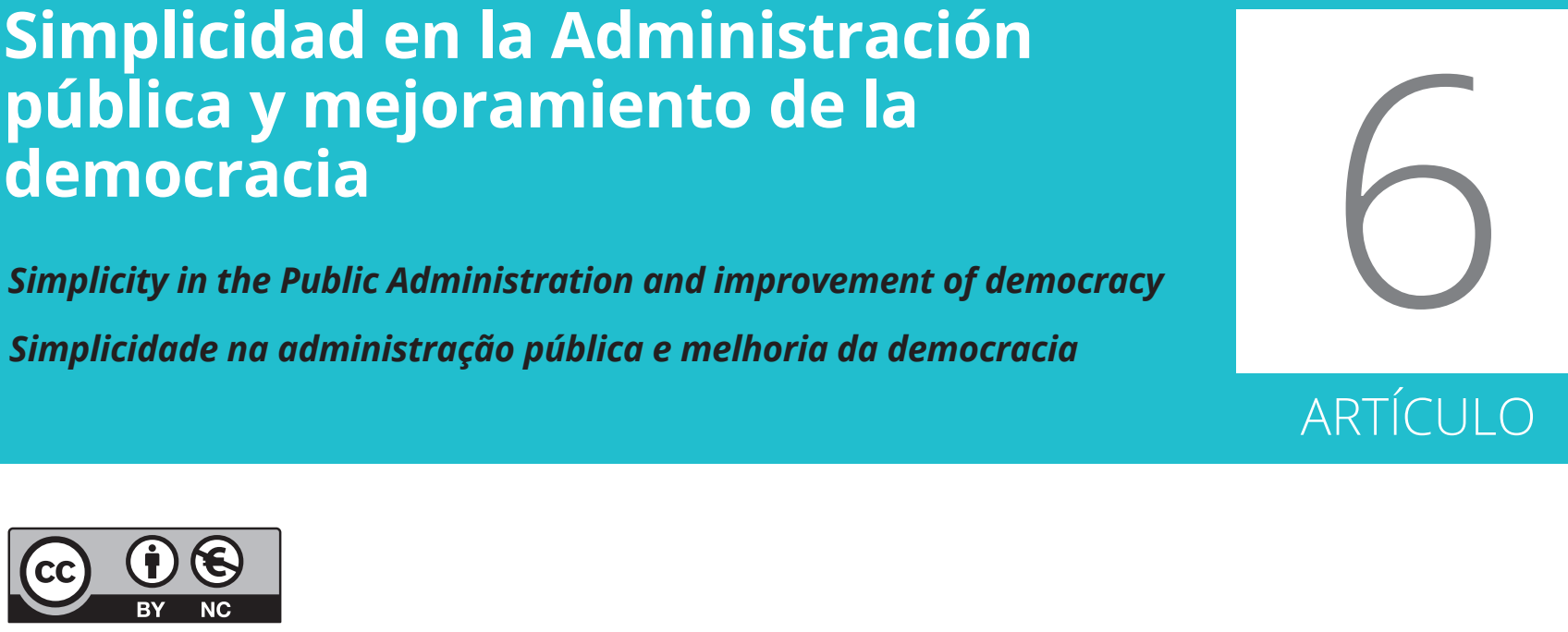

\section{Cristian Castillo Peñaherrera}

Universidad del Azuay (Ecuador)

Candidato a Doctor en Ciencias Políticas - Máster en Gobierno y Administración Pública Magister en Desarrollo y Comportamiento Organizacional - Licenciado en Comunicación Social. Docente investigador en gobierno, administración, simplificación administrativa, gestión organizacional. Ex Ministro de la Presidencia del Ecuador. Ex Ministro de la Administración Pública del Ecuador.

ccastillo@uazuay.edu.ec

orcid.org/0000-0003-0038-1483

RECIBIDO: 08 de junio de 2020 / ACEPTADO: 06 de agosto de 2020

\section{Resumen}

Este documento plantea que la democracia transita desde un modelo exclusivamente formal, hacia uno sustantivo cuando se da, entre otras condiciones, una reconfiguración de la Administración General del Estado. Una condición congruente es que esta se enfoque en proveer servicios públicos de calidad, usando los recursos tecnológicos disponibles actualmente para atender las expectativas y necesidades de la ciudadanía. El caso que se analiza es la experiencia del gobierno del Ecuador con su política pública de simplificación de trámites, y cómo esta iniciativa pudo incrementar la calidad de la democracia y facilitar condiciones para el desarrollo de una democracia sustantiva.

\section{PALABRAS CLAVE}

Democracia sustantiva, Simplificación administrativa, Servicios públicos digitales, Acceso a la administración.

\section{Abstract}

This article proposes that democracy moves from an exclusively formal model to a substantive one when, among other conditions, there is a reconfiguration of the General State Administration. A congruent condition is that it focuses on providing quality public services, using the 
technological resources currently available to meet the expectations and needs of citizens. The case being analyzed is the experience of the government of Ecuador with its public policy of simplifying procedures, and how this initiative was able to increase the quality of democracy and facilitate conditions for the development of a substantive democracy.

\section{KEYWORDS}

Substantive democracy, Administrative simplification, Digital public services, Access to the government.

\section{Resumo}

Este documento propõe que a democracia transita de um modelo exclusivamente formal para um substantivo quando, entre outras condições, há uma reconfiguração da Administração Geral do Estado. Uma condição consistente é que esta se concentre na prestação de serviços públicos de qualidade, usando os recursos tecnológicos atualmente disponíveis para atender às expectativas e necessidades dos cidadãos. O caso analisado é a experiência do governo do Equador com sua política pública de simplificação de procedimentos e como essa iniciativa foi capaz de aumentar a qualidade da democracia e facilitar as condições para o desenvolvimento de uma democracia substantiva.

\section{PALAVRAS-CHAVE}

Democracia substantiva, Simplificação administrativa, Serviços públicos digitais, Acesso à administração.

\section{INTRODUCCIÓN}

Una democracia transita desde un modelo formal hacia uno sustantivo cuando se dan acuerdos sociales (Quiroga, 2000) suficientes como para establecer que la democracia no solo debe garantizar las libertades individuales, sino también debería buscar aquello que, a falta de una forma mejor, se puede denominar, como el bien común, el cual, para los fines del presente documento, se entiende como la búsqueda constante de condiciones estructurales de igualdad real entre todos los miembros de esa sociedad.

Esta búsqueda sucede gracias a un conjunto de condiciones que incluyen, aunque no se limitan, a instituciones, tradición democrática y el rol del Estado en la configuración de esas bases estructurales. Otras condiciones necesarias tienen que ver con el fortalecimiento de la sociedad civil que viene a desplazar el "centro de gravedad en la relación entre los recursos que representan el dinero, el poder administrativo y la solidaridad" (Habermas, 2005, 8) de tal manera que se desarrollen, como sugiere el mismo autor, nuevos espacios públicos de formación democrática de la opinión y la voluntad política en el conjunto social y no en el sector privado o en el Estado. Esto re afirma a la sociedad frente al dinero o al poder administrativo.

En este estudio se propone que, para que la democracia pueda considerarse sustantiva, su calidad debería mejorar de manera sostenida y permanente. Para Jacobs (1994), la democracia no debe estar acotada a los procesos eleccionarios, sino que debe ser evidente entre cada proceso. El concepto de sustantivo se asocia, entre otras variables, a las que se relacionan con cómo instituciones políticas y públicas, dan resultados en la búsqueda de esas condiciones de igualdad real en el acceso a la garantía de derechos fundamentales (Spiker, 2007) y en el acceso real a las mismas oportunidades. 
Esto rompe una visión histórica del rol y funcionamiento de la Administración tradicional, tan dada a generar dificultades para vender facilidades (Correa R, comunicación personal, 2015). Se plantea una visión distinta, que se centra en la ciudadanía, en cómo debe operar para atender sus necesidades, cómo generar condiciones de competitividad del sector empresarial o facilitar el acceso a todos los procedimientos que se requieren para este fin.

El documento plantea varias reflexiones sobre esta forma de entender al Estado y a la Administración. En un primer momento, se hace un repaso a la noción del Estado moderno, superando la visión tradicional que le asigna la capacidad de monopolizar la violencia legítima. Posteriormente se proponen reflexiones sobre la democracia y su calidad, y cómo se puede entender sustantiva una democracia si el Estado y la Administración funcionan.

El artículo se ha construido sobre el análisis de información pública y publicada por el Gobierno y otros estudios relacionados, sobre la implementación de la política pública de simplificación de trámites en el periodo 2014-2016 en Ecuador. Como se podrá notar, se plantea que, a partir de la implementación de dicha política se facilitó a la ciudadanía el acceso a la Administración, lo que a su vez pudo mejorar la percepción de la ciudadanía sobre el funcionamiento del Estado y de la democracia.

En particular, la evidencia sugiere que el esfuerzo por aprovechar medios electrónicos pudo coadyuvar al fortalecimiento de la democracia sustantiva. La información que aporta el trabajo observa que la tramitación electrónica y la posibilidad de participar en los procesos de retroalimentación y mejoramiento de la calidad de los trámites y procedimientos administrativos que realiza la ciudadanía pudo alimentar condiciones sociales que permitieron configurar una democracia más sustantiva.

\section{LA NOCIÓN DEL ESTADO MODERNO}

Los Estados son la expresión más compleja de acuerdo social de una sociedad que se autorregula. El fin último del proceso democrático es decidir quién debe gobernar el Estado para dirigir la sociedad hacia lo que el conjunto social considera deseable. Se puede sugerir que no es posible hablar de calidad democrática si no existe un Estado institucionalizado que la sostenga. La calidad de la democracia no es posible sólo como un acuerdo de voluntades individuales, sino que requiere también de un aparataje institucional que le dé soporte. Este aparataje institucional está sostenido en el Estado, entendido como la suma de las relaciones de todos los actores sociales que en ella conviven. El Estado es, también y a la vez, consecuencia y origen de las acciones políticas que realizan los miembros de esa sociedad (Heller, 1998 [1934]).

Este Estado consolida su manifestación material en un conjunto interdependiente de instituciones que reúnen el poder y los recursos de la una coerción social y política (Oszlak, 2007) necesaria para la legitimidad de sus actos. La esfera en la que los grupos humanos se disputan el control del Estado es la esfera de lo político. Allí donde suceden las disputas dialécticas, ideológicas y programáticas que son posibles porque la sociedad ha aceptado que dichas disputas son necesarias para alimentar el proceso democrático (Levi, 2015 [2002]).

Es en el Estado en donde se desarrollan y cobran forma ideas como la de bienes públicos, sociedad justa o valores comunes (Bauman, 2002), ideas que tienen eco en el concepto de democracia sustantiva. Nociones que, además, requieren de una estructura de soporte que permita que la convivencia en dichas sociedades complejas haga posible la búsqueda de 
esos propósitos que aporta Bauman. La democracia sustantiva, entendida como la solución incluyente de los problemas públicos más relevantes para ese grupo social, que facilitan condiciones de igualdad real de oportunidades y que no se limitan solo al crecimiento económico (Giroux, 2005).

En este sentido, el rol del Estado "no es únicamente asegurar las condiciones para la acumulación de capital, sino también y, sobre todo, para el perfeccionamiento de la vida de sus ciudadanos en materia de derechos, de servicios y de políticas públicas" (Peña \& Lillo, 2018, p. 24). Por lo tanto, según el autor, se debe dejar de percibir al Estado como un mero reproductor de lo mercantil, puesto que su vocación es más amplia e incluye, entre otras cosas, la potestad de regular y dar forma a la vida en sociedad, para evitar la perennación de las desigualdades y desequilibrios que persisten en las sociedades. Este es uno de los caminos que consolidan la democracia. Estados fuertes, generan organizaciones públicas capaces, que prestan servicios de calidad a la ciudadanía.

En un efecto de espiral, las y los ciudadanos, al recibir mejores servicios, confía más en el Estado y podrían disponerse a participar de más procesos democráticos. Interesantemente, pueden decidir participar en los procesos que les permiten elegir a sus representantes, y en aquellos en los cuales co-construir soluciones a los problemas públicos de la sociedad, o exigir cuentas de las acciones de dichos representantes. En este sentido, se puede resumir que el Estado es "la mejor forma de lograr el orden social, de fomentar el crecimiento económico o de facilitar la expresión democrática" (Levi, 2015 [2002], p. 28).

En el periodo que se estudia en este artículo, el Estado ecuatoriano fue reformado con la intención de ampliar su presencia e incidencia en asuntos en que anteriormente, o no existía presencia estatal, o su incidencia era relativa o secundaria. Por ejemplo, el Gobierno creó el Ministerio de Minas, como una respuesta a la necesidad de darle más relevancia al sector, al considerar que el potencial minero que tiene el territorio nacional podía ser sujeto de aprovechamiento para la generación de nuevos sectores productivos, con el consiguiente incremento de ingresos para la sociedad. Otro ejemplo fue el énfasis asignado a la profesionalización de la gestión pública a través del fortalecimiento de la Secretaría Nacional de la Administración Pública -SNAP- como el organismo responsable de implementar procesos de gestión de la calidad, la implementación de gobierno electrónico, innovación o transparencia en la Administración central.

\section{CALIDAD DE LA DEMOCRACIA}

La sociedad organizada genera un Estado que actúa para resolver los problemas públicos complejos que de manera individual no podrán ser resueltos. Este Estado es gobernado por coaliciones que participan de los procesos democráticos para ganar el derecho a gobernar. En último término, la democracia formal tiene que ver con quién y cómo accede al poder, y cómo lo ejerce (Duhem, 2006). Es decir, cómo usa el poder para gobernar el Estado y resolver los problemas que la sociedad le ha encargado resolver. A partir de este momento, la democracia deja, o debería dejar, de ser formal exclusivamente, como quien arbitra el proceso de competencia y acceso al poder. En adelante, la democracia debería ser también sustantiva, como quien suscita condiciones para la construcción de sociedades de iguales, con igualdad de derechos e igualdad real de acceso a oportunidades. 
La democracia puede tener diferentes calidades relativas. El concepto de calidad de la democracia puede asociarse con 1) los procesos de participación democrática, 2) la forma en la que las instituciones políticas facilitan la consolidación de los procesos democráticos, permite la participación de todos los actores o generan condiciones de rendición de cuentas de las autoridades elegidas (Levine \& Molina, 2007). Otras corrientes reconocen, además, que la calidad de la democracia mejora 3) cuando funciona el Estado (PNUD-OEA,2010).

La participación democrática se entiende como el conjunto de acciones públicas que demuestran el rol que desempeñan las y los ciudadanos en los procesos de toma de decisiones de política pública. Estos procesos de deliberación societaria según Habermas (1995), validan las decisiones de la mayoría de los participantes en el marco de una discusión racional. Para esto, el proceso participativo debería apoyarse en la pluralidad de estilos de vida de las sociedades modernas (Velásquez, 2003) si es interés de la sociedad, elevar la calidad de la democracia.

La calidad de la democracia incluye la medida en que los funcionarios públicos están obligados a rendir cuentas a la ciudadanía sobre sus actos y decisiones en cuanto gobernantes (Levine \& Molina, 2007) y, en el mismo sentido, el grado en que el funcionamiento de las instituciones públicas, también está abierto al escrutinio público (Duhem, 2006). Nótese que no se trata solo de las acciones que realiza la coalición gobernante. Se trata también de que el aparataje estatal deba ser sujeto de escrutinio por parte de la sociedad en su conjunto. Por lo tanto, la democracia mejora si la ciudadanía siente que sus mandantes reportan sus acciones y las razones que las motivaron a tomarlas, y si es que el sistema de organizaciones públicas puede ser escrutado de forma que la gente se sienta informada y entienda las decisiones de política pública que toman tanto el gobierno como la administración ${ }^{1}$.

El Estado es uno de los principales ejecutores de la apertura democrática, ciudadana y participativa (Piñeiro, 2018). Debe tener capacidad, entre otras cosas, para canalizar las protestas legítimas de los sectores de la sociedad que se perciben a sí mismos como afectados por las decisiones políticas o por la operación de las organizaciones públicas; y, también, debería garantizar la presencia estable de un orden social que haga posible el ejercicio real de la libertad de la ciudadanía. Para esto, las organizaciones gubernamentales deberían operar en función de sus capacidades y responsabilidades, en la provisión de servicios públicos, acceso a bienes comunes o garantía de derechos, que permitan el ejercicio efectivo de dicha libertad.

En esta línea de pensamiento, organizaciones como el Programa de Naciones Unidas para el Desarrollo [PNUD], en conjunto con la Organización de Estados Americanos [OEA] (2010) plantean que las democracias se han empobrecido precisamente cuando sus Estados se han empobrecido o limitado, o en su defecto, siendo de mayor tamaño, no han estado al servicio de las mayorías.

Autores como Morlino (2005), o Duhem (2006) sostienen que la calidad de una democracia depende de una estructura institucional estable, que se sostiene, entre otras instituciones, en el Estado y su Administración, en la medida que estas operan para posibilitar esa libertad e igualdad reales de toda la ciudadanía. De lo cual podemos colegir que la calidad de la democracia aumenta si el Estado y sus institucio-

\footnotetext{
1 El Gobierno se entiende como la coalición que participó en elecciones y accedió al poder para dirigir el Estado. La Administración se entiende como el entramado de organizaciones públicas que materializan el Estado y que llevan a la práctica las decisiones del Gobierno.
} 
nes operan también con principios de calidad, transparencia, orientación a la ciudadanía, etc.

Se puede proponer también que el desempeño de la Administración refuerza la calidad de la democracia. Si las organizaciones que lo conforman son eficientes, dan servicios de calidad, tratan a toda la ciudadanía por igual, ésta puede percibir que, si las instituciones funcionan, la democracia funciona. Por lo tanto, será más propensa a participar de los procesos democráticos. De hecho, si las organizaciones del Estado operan de tal manera que sus acciones propendan a la construcción de una sociedad igualitaria, con condiciones dignas, cobertura de derechos y oportunidades reales para todos, la ciudadanía confiará en la democracia (Correa R, comunicación personal, 2015), y esta podría operar en clave sustantiva.

En el caso que se analiza, es interesante notar que, de acuerdo con Latinobarómetro (2020), la satisfacción de la sociedad ecuatoriana con la calidad de la democracia se incrementó de un 33,2\% en 2009, a un 51\% en 2017. En este mismo periodo, se puede verificar que el gobierno del Ecuador realizó reformas a las organizaciones públicas con el afán de mejorar la cobertura de los servicios públicos, implementar políticas de gestión de calidad, como la certificación de varios hospitales públicos con el certificado de calidad Accreditation Canada International - ACI (Ministerio de Salud Pública del Ecuador, 2014) o la obtención de la licencia de Bachillerato Internacional para más de 56 colegios públicos (Ministerio de Educación del Ecuador, 2015) o la puesta en marcha de un Plan Nacional de Simplificación de Trámites que abarcó a todas las entidades de la Administración central.

\section{ADMINISTRACIÓN PÚBLICA DE CALIDAD}

El Estado se materializa en un complejo entramado de organizaciones públicas que operan las diferentes funciones de ese Estado. El sistema de justicia se administra ${ }^{2}$ a partir de organizaciones públicas como los Consejos de la Judicatura que operan en algunos países. La Asamblea Nacional o el Congreso de los Diputados, también se administra a través de una organización claramente delimitada con personería jurídica y relativa autonomía financiera. La función Ejecutiva, en cuanto rectora del amplio espectro de políticas públicas que se implementan en un momento dado, actúa a través de un conjunto de organizaciones de gobierno (los ministerios cuando solo ejercen capacidad rectora y no prestan servicios directos) y organizaciones de administración instrumental (que prestan servicios públicos, garantizan acceso a derechos o cumplen con potestades estatales).

Las organizaciones en general surgen de la acción humana (Ayala Espino, 2001; Pérez, 1993), como respuesta a la necesidad de coordinación para alcanzar objetivos comunes o resolver problemas complejos. Pueden considerarse como un conjunto de posibilidades disponibles de solución a problemas para los que podrían ser la respuesta (Cohen et al., 2009[1972)). Adicionalmente, Ayala Espino (2001) plantea que las organizaciones públicas, además, proporcionan una estructura sobre la que se asientan determinadas interacciones sociales como los procesos educativos, el acceso a la justicia o el intercambio de bienes y servicios entre los miembros de la sociedad. El mismo autor su-

2 Administración en este contexto se entiende como la puesta en operación y la gestión de los recursos materiales, humanos, tecnológicos y financieros requeridos para su funcionamiento rutinario. 
giere, además, que las organizaciones públicas pueden ayudar a minimizar los costos derivados de ese intercambio, con lo cual las sociedades pueden tornarse más eficientes.

Mulas-Granados (2010) propone que el Estado de Bienestar debería evolucionar hacia un tipo diferente de Estado que sugiere llamarlo Dinamizador. Este nuevo tipo de Estado debería generar cambios permanentes en las sociedades en dos niveles diferentes, tanto en los medios y procedimientos, como en los objetivos y fines de su propia existencia. En este sentido, la Administración debería ser dinámica en su funcionamiento administrativo y dinamizadora en su relación con el resto de agentes económicos y sociales. En relación a los fines, las entidades públicas deberían generar condiciones para que se garantice la igualdad real de acceso a oportunidades para todas y todos los miembros de la sociedad que les permitan el pleno ejercicio de sus libertades individuales, como recalca Mulas-Granados (2010).

Esto demanda un cambio en el Estado y la Administración. Por ejemplo, esta debería estar en capacidad de anticiparse a los nuevos riesgos, demandas sociales, o demandas individuales y actuar de manera preventiva (Mulas - Granados, 2010). Todo lo opuesto al actuar reactivo que caracteriza a la Administración pública en general, cuando los riesgos ya están materializados. El gobierno de Corea denominó a esta aproximación como Government 3.0. y lo explica como un paradigma innovador "para la operación del gobierno, que busca proveer de servicios individualizados para la ciudadanía y el soporte a la creación de empleo en el sector creativo de la economía, a través de la apertura y compartición de información pública, así como también la eliminación de barreras de acceso dentro de la Administración" (Ministry of Security and Public Administration of Korea, 2013, p. 1)
Este ejercicio involucra cambios importantes en el enfoque tradicional de la gestión pública (Massal \& Sandoval, 2009). No se trata solo de lograr cambiar un trámite en sí mismo, sino de que toda la organización pública pueda entender la forma en la que la tecnología transforma sus relaciones internas y las relaciones con la ciudadanía. Un cambio de este tipo requiere esfuerzos relevantes en lo político, lo programático y lo instrumental de cualquier proyecto político. La evidencia sugiere que la tecnología y la calidad de la democracia tienen incidencia directa en los resultados de la acción de gobierno (Oriol, 2005), con lo cual se vuelve un imperativo institucional el fortalecimiento de las capacidades organizacionales para la incorporación de procesos tecnológicos que se traduzcan en el mejoramiento de la calidad en la entrega de servicios públicos y en la realización de procedimientos administrativos.

En 2014, el gobierno del Ecuador, a través de la SNAP (2015), lanzó dos planes nacionales orientados a reformar el enfoque de la Administración. Estos planes fueron: el Plan Nacional de Excelencia -PROEXCE- como una iniciativa para homogeneizar los proyectos de gestión de calidad al interior de la Administración central; y el Plan Nacional de Gobierno Electrónico -PNGE-, como una iniciativa para impulsar la tramitación electrónica de la mayor cantidad de procedimientos.

El PROEXCE se constituyó sobre la base del modelo de la European Foundation for Quality Management-EFQM-, aplicado a las administraciones públicas, denominado Common Asessment Framework -CAF- (Proaño, 2017; García Mejía et al., 2018) y sirvió de guía metodológica para todas las actividades de orientación de las organizaciones públicas, hacia la calidad. El plan ha tenido posteriores versiones, siendo la última, la expedida en 2018, por el Ministerio del Trabajo del Ecuador-MDT- $(2018,6)$ y en la que 
se señalan los siguientes objetivos: 1) Incrementar la calidad de los servicios públicos: 2) Incrementar la eficacia de la gestión de planes, programas, proyectos, servicios y procesos; y 3 ) Incrementar el nivel de madurez de la gestión institucional partiendo de los criterios del Modelo Ecuatoriano de Calidad y Excelencia.

EI PNGE (SNAP, 2014) por su parte, consolidó todas las iniciativas relativas a arquitectura, desarrollo de software y aplicaciones de la Administración para mejorar su eficiencia, incrementar la cantidad de procedimientos electrónicos y avanzar hacia una integración de bases de datos, sistemas operativos y demás actividades requeridas para una mejor prestación de servicios de parte de las organizaciones públicas, con el objetivo de mejorar la experiencia de la ciudadanía en el uso y acceso a plataformas electrónicas para realizar procedimientos administrativos de diversa índole.

Ambas iniciativas fueron el punto de partida de los esfuerzos de reconfiguración de la Administración pública del nivel central. Esto permitió que las organizaciones públicas implementen varias iniciativas de mejora de la calidad de la gestión en cada uno de sus ámbitos de acción y propendan al desarrollo de soluciones digitales que acerquen a la ciudadanía a la Administración.

\section{FACILITAR EL ACCESO A LA ADMINISTRACIÓN}

Facilitar el acceso a la Administración involucra la generación de condiciones estables y permanentes que hacen posible que todos los miembros de una sociedad puedan peticionar ante las autoridades, el acceso a determinados derechos o servicios públicos, o cumplir sus obligaciones con el Estado dadas las potestades establecidas en los marcos legales, sin que para ello tengan como única vía, que acudir a una ventanilla física ubicada en una sola área geográfica o tengan que hacerlo en condiciones que los afecten.

De acuerdo con la Organización para la Cooperación y el Desarrollo Económico [OCDE] (2006) la ciudadanía ha experimentado cambios en los años recientes, con más educación, mejor acceso a información, mayor capacidad crítica, que le hacen exigir servicios públicos de mejor calidad o procedimientos administrativos más simples y eficientes. A medida que las sociedades se vuelven más complejas, los costos de transacción al tener que interactuar con la Administración se vuelven más elevados, no solo en términos financieros (costos de tasas), sino también económicos (lucro cesante por no poder operar si no hay autorización estatal para una actividad económica) o sociales (tiempo destinado a la Administración que impide, por ejemplo, el cuidado de la familia).

Esta nueva realidad plantea nuevos retos para las organizaciones públicas, como bien señala la misma OCDE "el reto [...] será responder a las crecientes demandas de transacciones más eficientes, servicios a medida y acceso ubicuo por parte de ciudadanos y empresas" (2006, p. 56).

Cuando las organizaciones públicas deciden modificar el encuadre con el que operan para atender estas cambiantes y cada vez más complejas demandas, puede suceder que la sociedad refuerce la construcción de una ciudadanía responsable. En el sentido contrario, a la vez, un mejor acceso y democratización de la Administración puede redundar en el aumento de su eficiencia (Cunill, 2005). Esto puede incluir una forma de relacionamiento del Estado más orientada a satisfacer las necesidades y expectativas de la gente; es decir, más accesible (OCDE, 2006), y pueda generar más y mejores canales de atención de los requerimientos de las y los usuarios, procesos más sencillos de 
atención, eliminación de requisitos o procedimientos innecesarios, entre otras acciones que mejoren las oportunidades de acceso de la ciudadanía a la Administración, y a esta ser más eficiente en la prestación de los servicios o procedimientos que está obligada a ofrecer. Es de suponer que acciones como estas refuerzan la confianza de las y los habitantes en el Estado y el Gobierno, y esto a su vez, refuerza la calidad de la democracia.

Facilitar el acceso a la Administración puede ser beneficioso en dos sentidos (Brugué \& Gallego, 2001; OCDE, 2006). Por un lado, el Estado obtiene mejores niveles de aceptación y legitimación, lo que, a su vez, mejora la calidad de la democracia, pues la valida como forma de gobierno gracias a la legitimidad y credibilidad que se obtiene por ese mejoramiento. Por otro, instrumentalmente hablando, un mejor acceso mejora el rendimiento de las organizaciones públicas y puede ayudar a conseguir otros objetivos importantes de la política pública de la coalición gobernante, como el crecimiento económico y la cohesión social. En ambos casos, el acceso más sencillo a la Administración pública debiera alimentar la calidad de la democracia.

A medida que aumenta la complejidad de las sociedades, las Administraciones tienen que dejar de actuar en solitario (Brugué \& Gallego, 2001). Atrás va quedando la idea monopólica de la Administración tradicional, y en su lugar, se abre paso la intención cada vez más fuerte de consolidar la interacción estable y permanente entre la Administración contemporánea y la ciudadanía, lo que incluye la posibilidad de compartir actividades y diversificar la forma en la que estas suceden.

En 2013, el Presidente del Ecuador firmó el Decreto Ejecutivo 149 (Correa, 2013) con el cual emite disposiciones de obligatorio cumplimiento para toda la Administración pública del nivel central, en relación a un encuadre homogéneo que facilite la realización de las iniciativas de tramitación electrónica, así como orientar la atención a la ciudadanía con un enfoque de simplificación administrativa.

En este decreto se establece que las organizaciones públicas deberán, de manera obligatoria, facilitar la interacción de la ciudadanía u organizaciones privadas o de la sociedad civil con la Administración, lo cual involucra facilitar el acceso, tanto en la disminución de barreras, como en el incremento de canales de atención, el uso intensivo de tecnología y la interoperación entre bases de datos públicas, con lo cual deberían reducirse las cargas administrativas asociadas a la realización de todos estos procedimientos.

\section{LO SIMPLE COMO EXPRESIÓN DE DEMOCRACIA SUSTANTIVA}

Configurar una Administración pública al servicio de la ciudadanía obliga a reencuadrar la forma de relación tradicional que ha sido caracterizada como lenta, burocrática y nada colaborativa. La expresión más notoria de esta caracterización ha sido, históricamente, la realización de trámites. Y es que el trámite es "lo que conecta el ciudadano con el servicio, obligación o derecho público: no hay servicio de educación sin la inscripción; no hay servicio de salud sin la cita; y no hay pago de impuestos sin la remisión del formulario" (García Mejía et al., 2018, p. 12). En pocas palabras, no hay relación posible entre Estado y ciudadanía si no hay de por medio, un trámite.

Para atender a esa nueva ciudadanía sugerida por la OCDE, es pertinente lograr que la Administración se reinvente en todas sus formas, incluyendo la forma de resolver los trámites. Cuando se trata de la tramitología (Guzmán, 1999), tal vez sea necesario lograr que el trámi- 
te se convierta en un asunto de política pública, como se hizo en el caso ecuatoriano. Esta política fue priorizada al más alto nivel político, con un Consejero del Presidente Correa a la cabeza de la implementación de las estrategias de simplificación en la Administración del nivel central del gobierno. El propio Presidente Correa (2015) sugirió algunos principios importantes para esta política pública: 1) el mejor trámite es el que no se hace, 2) el Estado debe propender a disminuir los dolores de cabeza a la ciudadanía, 3) la mejor Administración es la que no molesta a la ciudadanía.

A partir de estos supuestos, se consolidó una estrategia de relacionamiento de las organizaciones públicas con la ciudadanía, a través de canales digitales, de manera que las y los habitantes del país puedan disminuir o eliminar la cantidad de veces que se desplazaban a una oficina pública para realizar un procedimiento administrativo o la cantidad de tiempo dedicado a realizar el procedimiento o la disminución de requisitos para realizarlos o, en ocasiones, todos los anteriores. Esta estrategia tuvo su momento más alto en los años 2015 y 2016, en los cuales se simplificaron un total de 446 trámites en 2015 y 410 en 2016 (García Mejía et al., 2018).

Los resultados obtenidos en ambos años se han documentado en publicaciones como la de García Mejía et al. (2018), y en reportes públicos como los que realizó el Presidente de la República en el espacio rutinario de rendición de cuentas denominado el Enlace Ciudadano³.

Como ejemplo se puede indicar que, en el año 2015, el número de requisitos por trámite se

3 Este espacio era un programa de televisión de carácter semanal en el que Correa informaba a la ciudadanía sobre diferentes asuntos de su acción de gobierno, y en algunas ocasiones se refirió a los avances en materia de simplificación administrativa redujo de 9 a 3, en promedio se evitó que 8 millones de personas acudan a una ventanilla física de atención, 40\% de los trámites simplificados tomaban menos de 4 horas para ser resueltos y la ciudadanía logró un ahorro de USD 20 millones, producto de la disminución de cargas administrativas innecesarias (CastiIlo, 2016). Para 2016, el número de requisitos bajó de 6 a 3, en promedio, los funcionarios destinaron un 57\% menos de horas a realizar procedimientos administrativos, producto de la automatización, y la ciudadanía redujo sus interacciones con la Administración en un 45\% por realización de trámites (García Mejía et al., 2018). En ambos años, se simplificaron un total de 856 procedimientos, de los cuales, 302 fueron simplificados a través de la automatización total. Esto quiere decir que, a partir de ese año, estos trámites se hicieron por medios digitales sin que medie la necesidad de acudir a una dependencia pública o se tenga que presentar documentación física de ningún tipo.

Esta mediación tecnológica es un espacio para la realización de transacciones de diversa índole. Es también, un medio de comunicación directo entre la ciudadanía y la Administración, toda vez que, en el proceso para acceder a la tramitación electrónica, las organizaciones públicas pudieron difundir mensajes relevantes sobre su sector y obtener retroalimentación de la ciudadanía sobre aspectos de interés sectorial.

Un ejemplo de esto fue la búsqueda de información que permita identificar aquellos trámites que pudieron representar mayores problemas para la ciudadanía, ya sea por la dificultad en acceder a ellos, o la cantidad de requisitos o pasos, o porque derechamente, no eran necesarios realizar. Para esto, el gobierno implementó la iniciativa de comunicación pública denominada Tramiton.to (www.tramiton.to) (García Mejía et al., 2018), que consistía en una 
plataforma digital en la cual las y los usuarios podían identificar los trámites que les ocasionaban problemas y sus propuestas de solución. La iniciativa fue presentada al público en junio de 2014 en uno de los Enlaces Ciudadanos indicados.

Para motivar la participación, la plataforma otorgaba premios a las mejores propuestas de simplificación realizadas por la ciudadanía, labor que además servía para mejorar la efectividad del plan, al retroalimentar desde la ciudadanía formas más adecuadas de hacer simples los procedimientos administrativos y empoderaba a las y los habitantes a participar en los mejoramientos de calidad de los servicios a los cuales acceden.

La iniciativa del Tramiton.to resultó ser un mecanismo de comunicación y participación ciudadana (Brugué \& Gallego, 2001) que consiguió llegar a las y los actores sociales interesados y/o afectados por la problemática de la tramitología. Adicionalmente, sirvió como fuente de información para identificar los trámites que requerían ser simplificados con más urgencia en cada uno de los años en los que la iniciativa estuvo en operación. La iniciativa recibió 6000 sugerencias provenientes de la ciudadanía para construir el Plan Nacional de Simplificación de Trámites 2016 (Barreiro, 2016). Esto mejoró la eficiencia de la herramienta de política pública, al focalizar los recursos y esfuerzos en aquellos trámites que fueron más sentidos por la ciudadanía participante.

Cuando la relación con la Administración se vuelve simple, el público tiende a confiar más en el Estado. Para que sea simple, es fundamental que se articulen, entre otras, dos acciones fundamentales. En primer lugar, la simplicidad de los trámites debe abarcar formas de redacción de las guías de los trámites en len- guaje llano, que evite tecnicismos que pueden tener sentido para las y los funcionarios que son los responsables del servicio, pero no necesariamente para la ciudadanía.

En segundo lugar, todo proceso de simplificación administrativa debe venir acompañada de procesos de alfabetización digital de las y los habitantes del territorio. De lo contrario, no se genera una verdadera democratización del Estado, sino todo lo opuesto. En el caso que nos ocupa, de la mano de los procesos de simplificación ejecutados desde el sector de la política pública de la administración pública, se implementaron iniciativas desde el sector de la política pública de la sociedad de la información que convirtió en gratuitas y de libre acceso a las redes wifi de los colegios públicos en varias zonas del país, y además implementó una red de 849 infocentros, oficinas públicas a las que podían acudir los habitantes de las zonas rurales para tener acceso a internet (en un modelo parecido al de un cibercafé) y en los que, un funcionario o funcionaria pública prestaban ayuda y facilitaban la tramitación electrónica cuando era requerido por la ciudadanía (Ministerio de Telecomunicaciones y de la Sociedad de la Información, 2018).

La simplicidad puede lograr que la ciudadanía se interese más por participar de otras iniciativas de solución de problemas que puedan reconfigurar las condiciones sociales y hacerlas menos inequitativas. De esto se puede colegir, que la democracia se refuerza, en el sentido de que la participación no es solo el acceso al sufragio, sino también a la relación con el Estado, en especial, cuando se busca aquello que al inicio denominamos, el bien común. 


\section{CONCLUSIONES}

La construcción de una sociedad democrática que trascienda la lógica de la democracia formal y acuerde consolidar un modelo de democracia sustantiva, en la que se disminuyan las inequidades y se construyan oportunidades de igualdad real para todas y todos, debería reformar sus instituciones en general para hacerlas inclusivas y al alcance de todas y todos. En particular, a lo largo de este artículo hemos sugerido que una de esas instituciones a reformar es el Estado.

La reforma del Estado debe propender a superar la lógica exclusiva de la protección de los derechos individuales, como los de propiedad, y debería migrar su mirada hacia la solución de aquellos problemas que por su complejidad y cómo afectan a la sociedad, se denominan públicos. Aquellos problemas que por su dimensión no pueden ser resueltos de manera individual, sino que requieren ser atendidos por el Estado, tanto en cuanto expresión de la sociedad en acción de Heller (1998[1934]).

Es importante reconfigurar la Administración pública para que deje atrás la visión tradicional de las organizaciones públicas como espacios de generación de problemas para la ciudadanía y, en su lugar, comprometerse con una visión proactiva de la acción pública que se anticipe a las necesidades de las y los ciudadanos de ese Estado y opere con criterios de eficiencia y efectividad, los cuales, en último término, deben entenderse como la generación de condiciones para la disminución de las inequidades sociales y la consolidación de los modelos de democracia sustantiva.

Una forma de acercar la Administración hacia esos deseables, es a través de condiciones que faciliten el acceso de todas y todos los usuarios en igualdad de condiciones, donde no se privilegie a los que habitan las ciudades, o los que tienen más recursos o los que tienen más contactos, sino, por el contrario, se amplían los canales, se eliminan barreras y se promueve el acceso directo sin intermediación para realizar los trámites que permiten, a las y los habitantes, acceder a servicios públicos, garantizar derechos, o cumplir responsabilidades ciudadanas.

Es recomendable que los trámites se sujeten a procesos de simplificación que prioricen el uso de canales digitales que universalicen el acceso de la ciudadanía a la Administración. No solo eso, el gobierno puede proponer iniciativas de retroalimentación y participación ciudadana como el Tramitón.to en Ecuador que permitió que las y los interesados puedan proponer y proveer de información sobre cuáles deben ser los procedimientos administrativos que requieren de mayor atención para hacerlos más sencillos.

La implementación de varias políticas públicas de gestión de la cosa pública coincide en el tiempo con los resultados de mejoramiento de la satisfacción de la ciudadanía con la calidad de la democracia, lo que puede sugerir que, si la ciudadanía verifica que la Administración General del Estado funciona mejor, amplía sus canales de atención, promueve la sencillez en la prestación de servicios públicos, hace más simples las tramitaciones al aprovechar los canales digitales disponibles y crear nuevos, puede devenir en un mayor interés de las y los ciudadanos por proponer mejoras a los sistemas de gestión y administración de las organizaciones públicas, en especial aquellas que disminuyan las condiciones de desigualdad estructural que persisten en sociedades como las latinoamericanas. 


\section{REFERENCIAS}

Ayala Espino, J. (2001). Instituciones y economía: una introducción al neoinstitucionalismo económico. Région y Sociedad, 13(22), 194-197.

Barreiro, G. (2016, agosto 17). 'Tramitón': Un portal digital que simplifica trámites a los ecuatorianos. Notimundo Hoy. https://notimundo.com.ec/ tramiton-un-portal-digital-que-simplifica-tramites-a-los-ecuatorianos/

Bauman, Z. (2002). En Busca de la Política. Fondo de Cultura Económica.

Brugué, J., \& Gallego, Q. (2001). ¿Una Administración Pública Democrática? En J. Font (Ed.), Ciudadanos y decisiones públicas. Ariel Ciencia Política.

Castillo, C. (2016). Plan Nacional de Simplificación de Trámites 2016. Presentación en Gabinete Ampliado Del Gobierno Del Presidente Rafael Correa Delgado. Working paper. Recuperado el 5 de junio de 2020 de: https://www.researchgate.net/publication/341946423_Plan_Nacional_ de_Simplificacion_de_Tramites_-PNST_2016

Cohen, M. D., March, J. G., \& Olsen, J. P. (2009). A garbage can model of organizational choice. Change Management, 229-262. http://www.econis. eu/PPNSET?PPN=604908822

Decreto Ejecutivo de Gobierno Electrónico y Simplificación de Trámites, Decreto U.S.C. (2013). www.gobiernoelectronico.gob.ec>uploadsı2018/08

Correa, R. (2015). En Castillo C. (Ed.), Retroalimentación al Plan Nacional de Simplificación de Trámites. Comunicación personal. Marzo de 2015.

Cunill, N. (2005). La democratización de la administración pública. Los mitos a vencer. Observatorio De La Corrupción E Impunidad, https://oci.juridicas. unam.mx/detalle-evidencias/72/la-democratizaci\%C3\%B3n-de-la-administraci\%C3\%B3n-p\%C3\%BAblica.-los-mitos-a-vencer

Duhem, M. (2006). La importancia de definir un concepto de calidad de la democracia. El cotidiano, 21(140), 58-66.

García Mejía, M., Molina, A., Reyes, A., \& Roseth, B. (Eds.). (2018). Construyendo un Estado orientado al ciudadano. BID.

Giroux, H., (2005). Neoliberalismo y crisis de la democracia. Anales de la educación común. Tercer Siglo - Año 1 - Número 1-2. Recuperado el 13 de julio de: http://isfdluisfleloir.edu.ar/wp-content/uploads/2013/03/ NEOLIBERALISMO-Y-CRISIS-DE-DEMOCRACIA.pdf

Guzmán, A., (1999, octubre 26-28). Tramitología, simplificación de procesos administrativos y reingeniería de negocios. [Ponencia]. II Congreso Internacional de la Mujer. México DF. México.

Habermas, J. (1995). Between facts and norms. MIT Press. 
Habermas, J. (2005) "Tres modelos de democracia. Sobre el concepto de una política deliberativa", Polis [En línea], URL: http://journals.openedition. org/polis/7473

Heller, H. (1998[1934]). Teoría del Estado. Fondo de Cultura Económica.

Jacobs, L., \& Shapiro, R. (1994). Studying Substantive Democracy. PS: Political Science and Politics, 27(1), 9-17. http://dx.doi.org/10.2307/420450

Latinobarómetro. (2020). Resultados Latinobarómetro Ecuador. Recuperado el 5 de junio de 2020 en http://www.latinobarometro.org/latCodebooks. jsp

Levi, M. (2015[2002]). El estado del estudio del Estado. In CAF (Ed.), El valor estratégico de la gestión pública (pp. 25-65). CAF.

Levine, D. H., \& Molina, J. E. (2007), La calidad de la democracia en América Latina: una visión comparada. América Latina Hoy, (45), 17-46.

Massal, J., \& Sandoval, C. (2010). Gobierno electrónico. ¿Estado, ciudadanía y democracia en Internet? Análisis Político, 23(68), 3-25.

Ministerio Del Trabajo del Ecuador. (2018). Guía Metodológica De Aplicación Del Modelo Ecuatoriano De Calidad Y Excelencia. Quito. Recuperado el 5 de junio de 2020 de: www.trabajo.gob.ecıuploadsıdownloads 2018/08

Ministerio De Educación del Ecuador. (2015). Ecuador celebra la acreditación de 56 colegios públicos al Programa de Diploma de Bachillerato Internacional. Recuperado el 5 de junio de 2020 de: https://educacion.gob.ec/ ecuador-celebra-la-acreditacion-de-56-colegios-publicos-al-programa-de-diploma-de-bachillerato-internacional/

Ministerio de Salud Pública del Ecuador. (2014). Accreditation Canada International felicita a Ecuador por el proceso de acreditación emprendido en Ecuador. Recuperado el 5 de junio de 2020 de: https://www.salud.gob. ec/accreditation-canada-international-felicita-a-ecuador-por-el-proceso-de-acreditacion-emprendido-en-ecuador/

Ministerio de Telecomunicaciones y de Sociedad de la Información del Ecuador. (2018). Proyecto Ampliación de la Red de Infocentros. Recuperado el 10 de julio de 2020 de: https://www.telecomunicaciones.gob.ec/ wp-content/uploads/2019/02/Infocentros-Documento.pdf

Ministry of Security and Public Administration of Korea. (2013). Government 3.0. Seúl

Morlino, L. (2005). Calidad de la Democracia, Notas para discusión. Metapolítica, (39), 37-53.

Mulas-Granados, C. (2010). El Estado Dinamizador: De los nuevos riesgos a las nuevas políticas. En C. Mulas-Granados (Ed.), El Estado Dinamizador: Nuevos Riesgos, Nuevas Políticas y la Reforma del Estado de Bienestar en Europa (pp. 53-69). Editorial Complutense. 
OCDE. (2006). La modernización del Estado: el camino a seguir. Instituto Nacional de Administración Pública.

Oriol Prats, J. (2005). Nuevas tecnologías, democracia y eficiencia de las instituciones políticas. Papers. Revista de Sociologia, 78, 151-168. Recuperado el 13 de julio de 2020 de http://dx.doi.org/10.5565/rev/papers/ v78n0.892

Oszlak, O. (2007). Formación histórica del Estado en América latina: elementos teórico-metodológicos para su estudio. En C. Acuña (Ed.), Lecturas sobre el Estado y las políticas públicas: Retomando el debate de ayer para fortalecer el actual (pp. 12-37). Centro de Estudios de Estado y Sociedad.

Peña y Lillo, J. (2018). El Estado en disputa frente a la contraofensiva neoliberal. CIESPAL.

Pérez, J. A. (1993). Fundamentos de la dirección de empresas. Ediciones Rialp.

Piñeiro Aguiar, E. (2018). El Estado ritual: imaginarios, mitos y defensa de lo político común. En J. Peña y Lillo, \& J. Polo Blanco (Eds.), El Estado en disputa frente a la contraofensiva neoliberal (pp. 65-88). CIESPAL.

PNUD, \& OEA. (2010). Nuestra Democracia. Fondo de Cultura Económica.

Proaño, J. (2017). Evaluación del Modelo Ecuatoriano de Excelencia y su aplicación en la Agencia Nacional de Tránsito en el periodo 2014-2015 [Tesis de maestría, Facultad de Ciencias Administrativas de la Escuela Politécnica Nacional]. Biblioteca Escuela Politécnica Nacional http://bibdigital. epn.edu.ec/handle/15000/17256

Quiroga, H. (2000). ¿Democracia procedimental o democracia sustantiva? La opción por un modelo de integración. Revista de Ciencias Sociales, 6(3), 361-374.

SNAP. (2014). Plan Nacional de Gobierno Electrónico

SNAP. (2015). Modelo R PROEXCE. Working Paper en Modelo R PROEXCE

Spicker, P. (2007) Government for the people: the substantive elements of democracy. International Journal of Social Welfare

Velásquez, F. (2003). ¿Qué ha pasado con la participación ciudadana en Colombia? Fundación Corona. 